\title{
Study of fracture mechanisms of multiphase polymers using the double-notch four-point-bending method
}

\author{
H.-J.SUE*, A. F. YEE \\ Macromolecular Research Center, Department of Materials Science and Engineering, \\ University of Michigan, Ann Arbor, MI 48109, USA
}

The double-notch four-point-bend technique (DN-4PB) is developed to study the fracture mechanisms of multiphase polymers. This technique is found to be effective for an unambiguous determination of the fracture mechanisms and the sequence of toughening events of polymer alloys when fracture occurs. The DN-4PB technique is also found to be especially useful for situations where the quantity of the test material is limited and the testing rate is high. The present study demonstrates the usefulness of the DN-4PB technique in a variety of polymeric systems and testing conditions. Requirements for which the DN-4PB technique becomes useful are also addressed.

\section{Introduction}

Toughening and strengthening of high-performance plastics for structural and aerospace applications has been a major area for research and development efforts in both industrial and academic arenas for decades [1-21]. However, detailed fracture mechanisms and the role(s) that the toughener or filler phase plays in the crack growth process remain unsettled in some cases. As a result, trial and error is still the prevalent practice for developing toughened plastics. A precise methodology for designing toughened polymer alloys is still beyond reach.

In order to avoid the time-consuming, and sometimes misleading, trial and error approach for developing toughened polymer alloys and filled plastics, it is imperative that one fully understands the fundamental parameters that govern their fracture. Specifically, the sequence of damage or toughening events and the role(s) the toughener or filler phase plays with respect to each operative microscopic mechanism(s) have to be known when polymers fracture. To achieve these goals, unambiguous and effective methods have to be utilized to reveal the crack evolution process of these plastics.

The most common approaches used to determine the micromechanical deformation mechanisms involves the inspection of completely failed specimens using scanning electron microscopy (SEM), optical microscopy $(\mathrm{OM})$ and transmission electron microscopy (TEM) techniques $[2,16,19]$. Studies of the fracture behaviour of plastics solely relying on fracture surface observations using SEM and/or reflected OM usually result in gaining either an incomplete understanding or misleading information concerning how exactly the polymer fails $[4,5,18,19,21]$. The invest- igation of a completely failed specimen with the damage left in the wake of a propagating crack, using $\mathrm{OM}$ and/or TEM, often does not enable one to discern which mechanisms are responsible for the formation and failure of the process zone $[2,3,19]$. Tensile dilatometry has sometimes been used to delineate the sequence and types of deformation mechanism [6], but the low hydrostatic stress component and the low rate achievable limit the applicability of these observations $[5,18]$.

The purpose of this paper is to describe the adaptation to polymers of a technique used to probe the micromechanical deformation mechanisms in metals and ceramics. This technique is known as the doublenotch four-point-bend (DN-4PB) method. Actually it has already been proved to be useful in deducing the deformation mechanisms in polyamide-6,6/polyphenylene oxide (PA-PPO) blends and other rubbermodified plastics $[4,5,18,19,21,22]$. The principle of this technique is rather simple: two nearly identical cracks are cut into the same edge of a rectangular beam. This beam is loaded in a four-point bending geometry with the cracks positioned on the tensile side (Figs 1 and 2). The portion of the beam between the two inner loading points is subjected to a constant bending moment. Thus the two cracks experience nearly identical stresses. As load is applied, plastic zones form in front of the crack tips. Since the two cracks cannot be exactly identical, one crack will propagate unstably, leaving behind the other crack with a nearly critically developed process zone at its tip. Since this crack is arrested, the events in the crack tip process zone are not obliterated by the last process, which in ductile plastics often involves tearing of the plastic ligaments spanning the crack faces. Various 

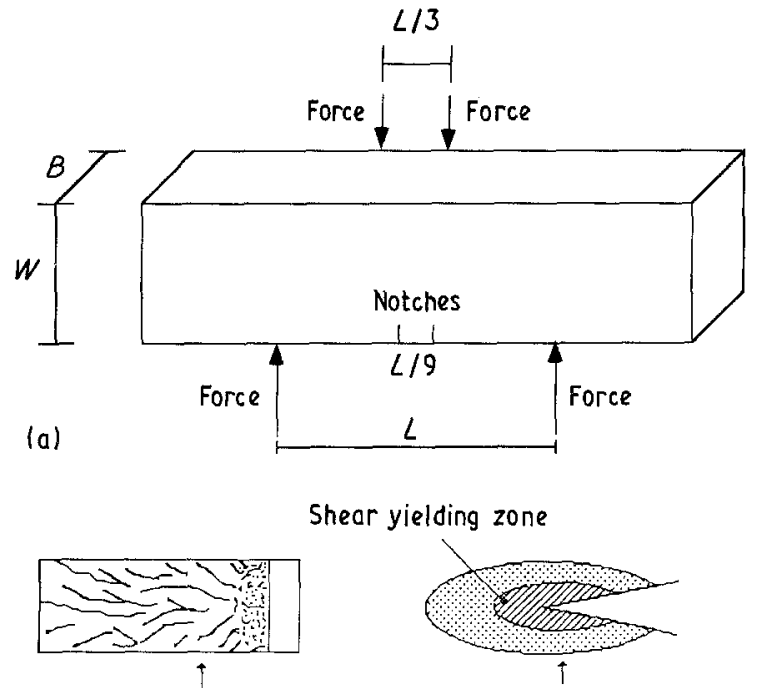

Shear yielding zone

Frocture surface

(b)

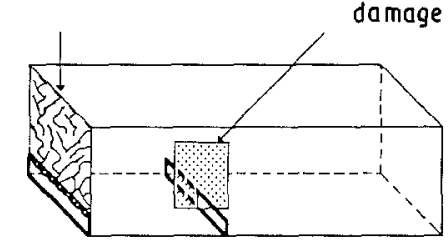

Figure $l$ (a) Schematic diagram of the DN-4PB geometry; $L$ $=7.62 \mathrm{~cm}, W=1.27 \mathrm{~cm}, B=0.635 \mathrm{~cm}$. (b) Types of damage generated in the DN-4PB specimen.

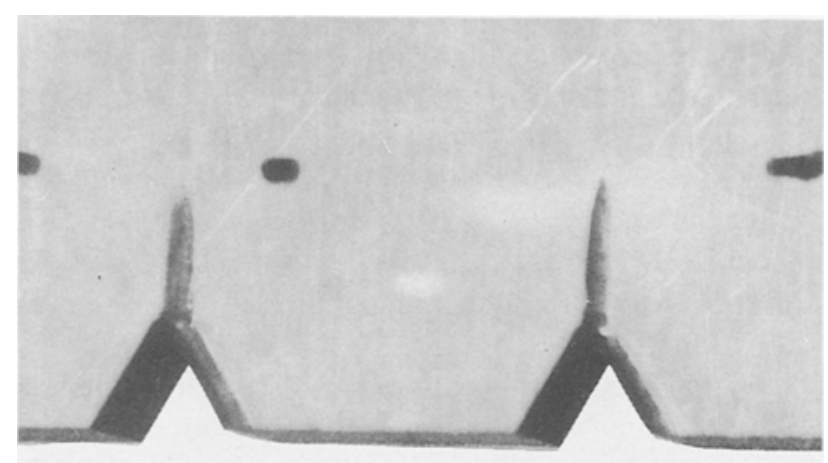

Figure 2 Reflected-light micrograph showing the two nearly identical sharp cracks within the two inner loading points for a DN-4PB test.

thin-sectioning and microscopy techniques can then be applied to probe those deformation mechanisms which occur at the crack tip and its wake (Fig. 1b).

To illustrate the usefulness of the DN-4PB technique, we will examine the process zones produced in a variety of toughened polycarbonates (PC) under various testing conditions. This technique is also useful for studying the fracture mechanisms of filled polymers, as will be obvious from the results presented here.

\section{Experimental procedure}

Polycarbonate $(\mathrm{MW}=26000)$ was blended in a Brabender with $3.3 \mathrm{wt} \%$ of a toughener phase. Two core-shell rubbers (CSRs), received in dry powder form, were used to toughen PC: Acryloid KM 653 particles which contain an MBS rubber (PMMA shell and polybutadiene-styrene core), and Acryloid KM 330 particles which contain an acrylic rubber (PMMA shell and polybutylacrylate core). Polycarbonate was also blended with $3.3 \mathrm{wt} \%$ polyethylene $(\mathrm{PE})$ in order to examine the role of adhesion in the toughening mechanism(s). All three tougheners were known to be effective in reducing the notch sensitivity of PC [5]. The PC blends were then compression-moulded into $6.35 \mathrm{~mm}$ thick plaques.

The DN-4PB test specimens were cut from the compression moulded toughened $\mathrm{PC}$ plaques into rectangular bars $(76.2 \mathrm{~mm} \times 12.7 \mathrm{~mm} \times 6.35 \mathrm{~mm})$ and pre-notched using a jeweller's saw, and precracked using a liquid nitrogen-chilled razor blade (Fig. 2). The DN-4PB specimens were then tested using a servohydraulic testing machine (Instron model 1331), at several crosshead speeds ranging from 0.0508 to $0.254 \mathrm{~m} \mathrm{~s}^{-1}$. The specimens rested on a fixture which had the outer loading points. The inner loading points were connected to the load cell in the testing machine. The lower fixture was raised by the hydraulic actuator until the upper loading points came into contact with the specimen. To ensure that the inner loading points contacted the DN-4PB specimens simultaneously, the stress-induced birefringence patterns at the two cracks of a loaded pure PC specimen were monitored with polarized light (Fig. 3). As shown in Fig. 3, the birefringence patterns induced at the two crack tips were found to be nearly identical. We concluded that the two cracks were experiencing nearly identical stresses.

The fracture surfaces of the failed specimens were cut to an appropriate size and coated with a $20 \mathrm{~nm}$ layer of Au-Pd for SEM (Hitachi S-520) studies.

The sub-fracture surface zone (SFSZ) was probed using two techniques. The first one involved petrographic polishing to the mid-plane of the specimen (Fig. 1b) and subsequent examination using a microscope in transmitted light. The second technique

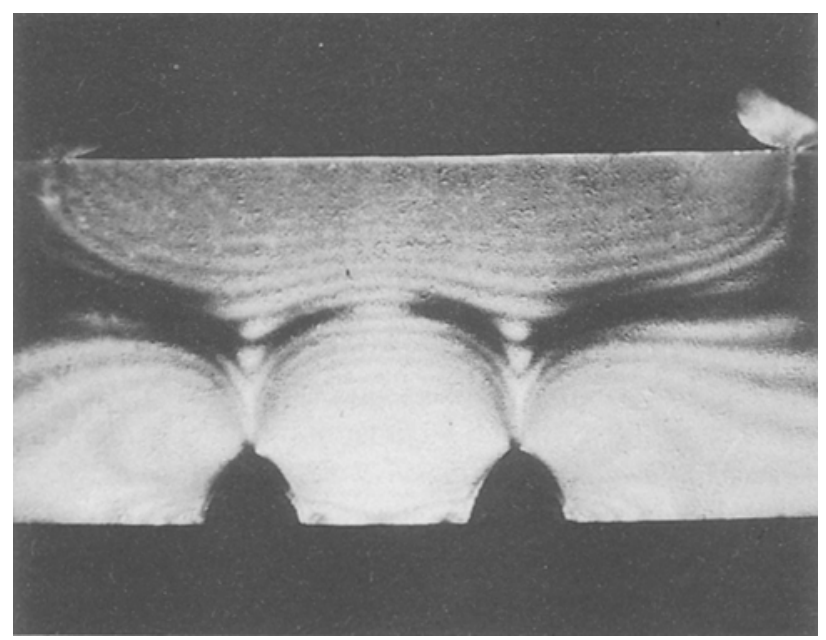

Figure 3 Transmitted-light micrograph, under crossed polars, showing the nearly identical stress-induced birefringence around the two cracks. 
involved microtomy. The blocks that contained the crack tip process zone were cut and polished to remove the skin region $[5,18,21]$. The remaining cores were then stained with a $2 \%$ aqueous solution of $\mathrm{OsO}_{4}$ for about $48 \mathrm{~h}$. Afterwards, these blocks were microtomed using a Reichert Ultracut E43 to make thin sections of the order of $0.1 \mu \mathrm{m}$ in thickness. The thin sections were cut parallel to the crack direction, but perpendicular to the fracture surface. They were laid on top of copper grids and examined using a Zeiss EM-10A (or Philips 400) TEM operating at an accelerating voltage of $100 \mathrm{kV}$.

\section{Results and discussion}

The present work is primarily intended to demonstrate the usefulness of the DN-4PB method for probing the toughening mechanisms of toughened plastics. The detailed failure mechanisms and sequence of failure events will be discussed in separate papers. Conditions under which the DN-4PB technique becomes useful are discussed in detail.

SEM is among the most frequently utilized techniques for studying the fracture behaviour of polymers due to its ease of use. However, only fracture events
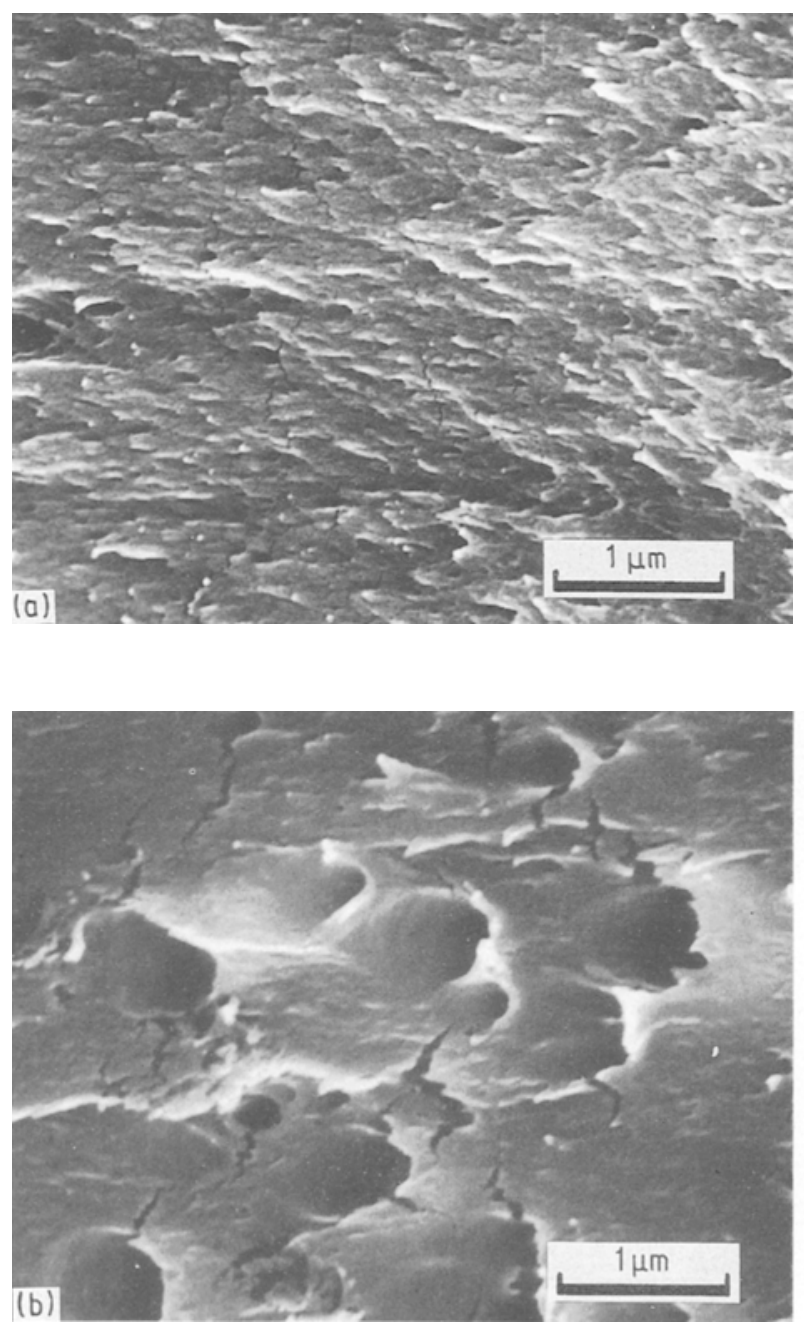

that evolve from the fracture surface can be detected using SEM. The failure events beneath the fracture surface generally cannot be observed. Furthermore, the role the toughener particles play during the fracture process cannot be understood via SEM observations. To demonstrate these points, the fracture surfaces of the KM 653, KM 330 and PE toughened PC systems are studied using SEM. Investigations of the fracture surfaces (Fig. 4) reveal evidence for cavitation and/or debonding of the toughener particles and ductile plastic deformation of the matrix. However, these micrographs give little hint as to what processes might have occurred in the SFSZ. Information concerning the size and shape of the process zones, which is an approximate albeit quite accurate indication of the toughening effect, among the three toughened systems cannot be determined. Further, the role(s) the toughener phases play upon fracture cannot be revealed. Therefore, it is not possible to discern any different roles the KM 653, KM 330 and PE toughener particles might have played during the fracture process.

In contrast, when the crack tip SFSZ of the KM 653, KM 330 and PE toughened PC systems generated from the DN-4PB tests are studied using OM, the differences among the three toughened $\mathrm{PCs}$ are readily apparent (Fig. 5). The bright-field OM micrographs shown in Fig. 5 reveal differences in the size and shape of the process zones (note the differences in magnification.) These differences imply that the toughener phases, i.e. PE, KM 653 and KM 330, must have behaved somewhat differently in toughening the $\mathrm{PC}$ $[5,20,23]$. The detailed failure mechanisms of these three systems, which require the use of TEM, will be discussed in separate papers. For illustration, TEM investigations on the toughening mechanisms of $\mathrm{PC}$ modified with KM 653 are described and discussed below (see also $[5,18,20,21]$ ).

Since the KM 653 core-shell particles contain an unsaturated butadiene rubber core, they can be easily stained with $\mathrm{OsO}_{4}$. The $\mathrm{OsO}_{4}$ staining of the butadiene rubber core helps to (i) harden the butadiene

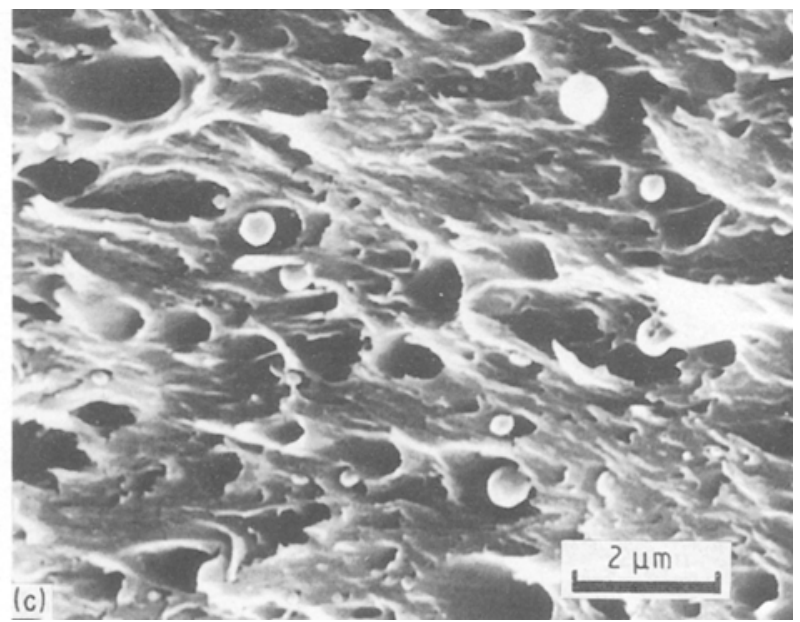

Figure 4 SEM micrographs taken on the fracture surfaces of (a) PC-KM 653, (b) PC-KM 330 and (c) PC-PE toughened systems. Only plastic flow of the matrix and holes induced by the toughener particles are observed on the fracture surface (note the differences in magnification). The crack propagates from right to left. 

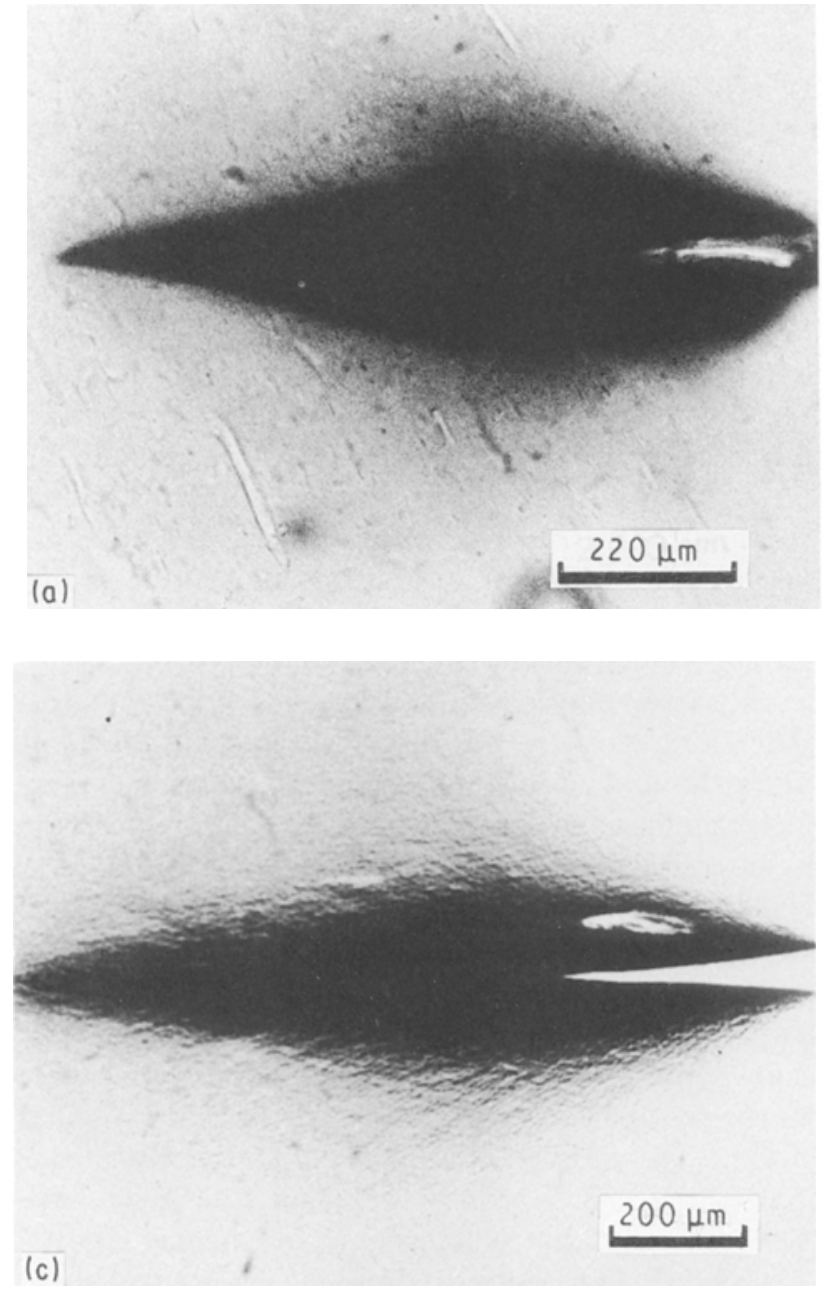

rubber core, which reduces the extent of smearing and pull-out of the rubber particles during microtomy, and (ii) induce sufficient phase contrast for distinguishing the rubber particles from the PC matrix. Fig. 6 shows TEM micrographs taken at the crack tip of a PC-KM $653 \mathrm{DN}-4 \mathrm{~PB}$ specimen. Cavitation and shearing of the rubber particles are found. In the crack wake, cavitation and shearing of the particles are also observed (Fig. 7). The size of the cavities inside the rubber particles diminishes in size with distance of the rubber particles from the crack. The shearing of the rubber particles becomes more severe toward the crack. We surmise that the matrix around the sheared rubber particles must have plastically deformed to a similar degree. In the region ahead of the crack tip, these rubber particles had actually cavitated prior to being distorted by subsequent shear deformation. In fact, these cavitated rubber particles are so highly deformed that they appear to have closed up due to the shearing process $[18,21,24]$. These, along with the OM observation (Fig. 5a), make it clear that cavitation of rubber particles (i.e. the grey zone that surrounds the dark shear-yielded zone) precedes the formation of the shear-yielded zone. In other words, when the matrix material around the crack tip is loaded in the opening mode (i.e. mode I fracture), rubber particle cavitation occurs first. The cavitation of the rubber particles helps to relieve the crack tip plane strain

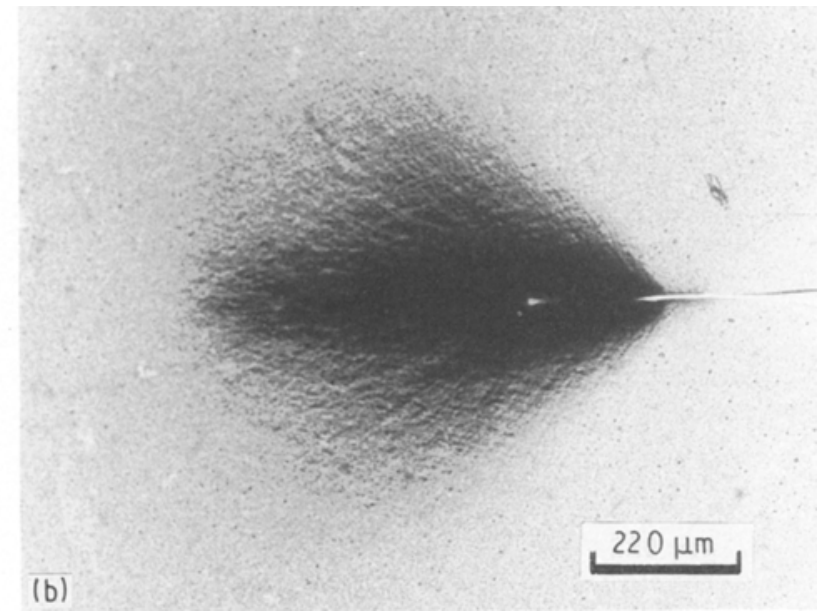

Figure 5 Bright-field transmitted light micrographs of the DN-4PB SFSZ of (a) PC-KM 653, (b) PC-KM 330 and (c) PC-PE toughened systems. It is clear that the damage zone sizes and shapes among these systems are quite different (note the differences in magnification). The crack propagates from right to left.

constraint and therefore facilitate the formation of shear yielding. This observation is consistent with our previous postulate that the triaxial tension in front of the crack tip has to be relieved (via rubber particle cavitation) in order for the formation of the shearyielded zone to become possible [1].

The above examples demonstrate that the DN-4PB method is useful for the unambiguous determination of failure mechanisms of toughened polymers under quasi-static loadings. The DN-4PB method is also found to be particularly valuable for studying the crack tip SFSZ of toughened polymers when the testing rate is high. The generation of a subcritically propagated crack under high testing rates ( $>1 \mathrm{mmin}^{-1}$ ) is found to be virtually impossible when using conventional specimen geometries [25] and testing machines. In an effort to demonstrate the usefulness of the DN-4PB technique for relatively high testing rates, the servohydraulic testing machine was programmed to move at a speed of $0.254 \mathrm{~m} \mathrm{~s}^{-1}$. This generated a dynamic loading condition on a PC-KM $330 \mathrm{DN}-4 \mathrm{~PB}$ specimen. The result of such a test is shown in Fig. 8. The crack tip damage zone of the KM 330-modified PC shows the formation of large-scale radial crazes/cracks around the damage zone. The pattern of these radial crazes/cracks resembles that found around fractured polystyrene which is due to crazing [26]. The formation of the radial crazes/cracks in front of the crack tip, which has not been observed under quasi-static loading conditions (e.g. Fig. 5b), is probably due to the dynamic loading effect. The toughening mechanisms due to dynamic loading can then be definitively studied using appropriate microscopy tools. This type of impact damage information cannot be obtained from any other conventional specimen or testing set-up. We believe the DN-4PB technique is ideal for studying impact fracture mechanisms of toughened polymers in both Izod and Charpy geometries. With minor modifications this technique 

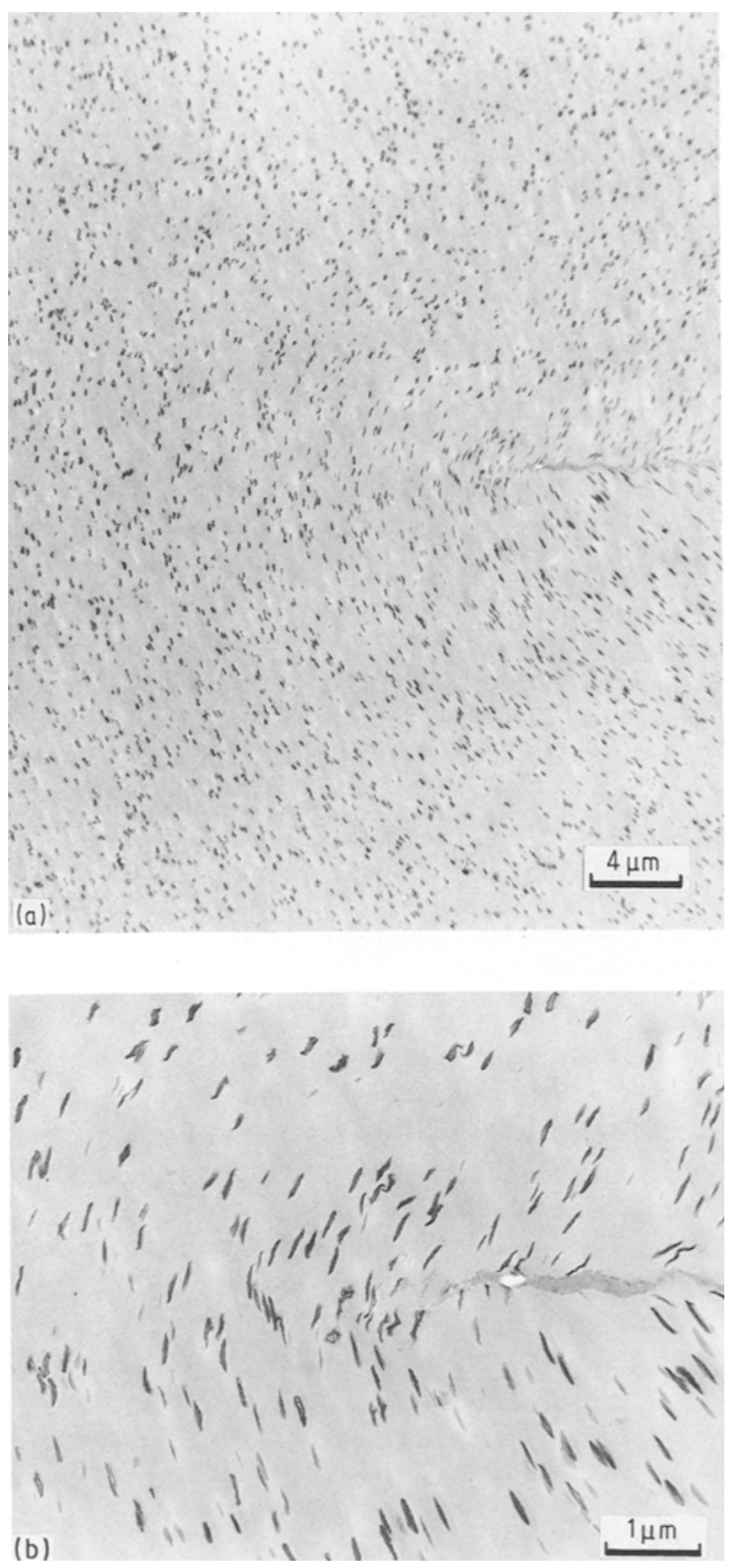

Figure 6 TEM micrographs taken at (a) the DN-4PB crack tip of the PC-KM 653 system and (b) a higher magnification of (a). Large elongation and cavitation of the rubber particles are observed around the crack tip region. The crack propagates from right to left

could also be used to study tensile impact. This will, in turn, help develop better tougheners for polymers.

The present paper has demonstrated the usefulness of the DN-4PB technique for studying the failure mechanisms of toughened plastics. It is noted, however, that care must be taken to ensure the generation of two nearly identical cracks between the two inner loading points of the DN-4PB geometry. It has been shown that if the material is quite tough (e.g., $K_{\mathrm{Ic}}>$ 1.2 $\mathrm{MPa} \mathrm{m}^{0.5}$ ), the subcritical crack growth of the surviving DN-4PB crack can be rather insensitive to the differences between the two crack lengths $[22,27]$. On the other hand, if the material is brittle, it is found that the differences between the two crack lengths

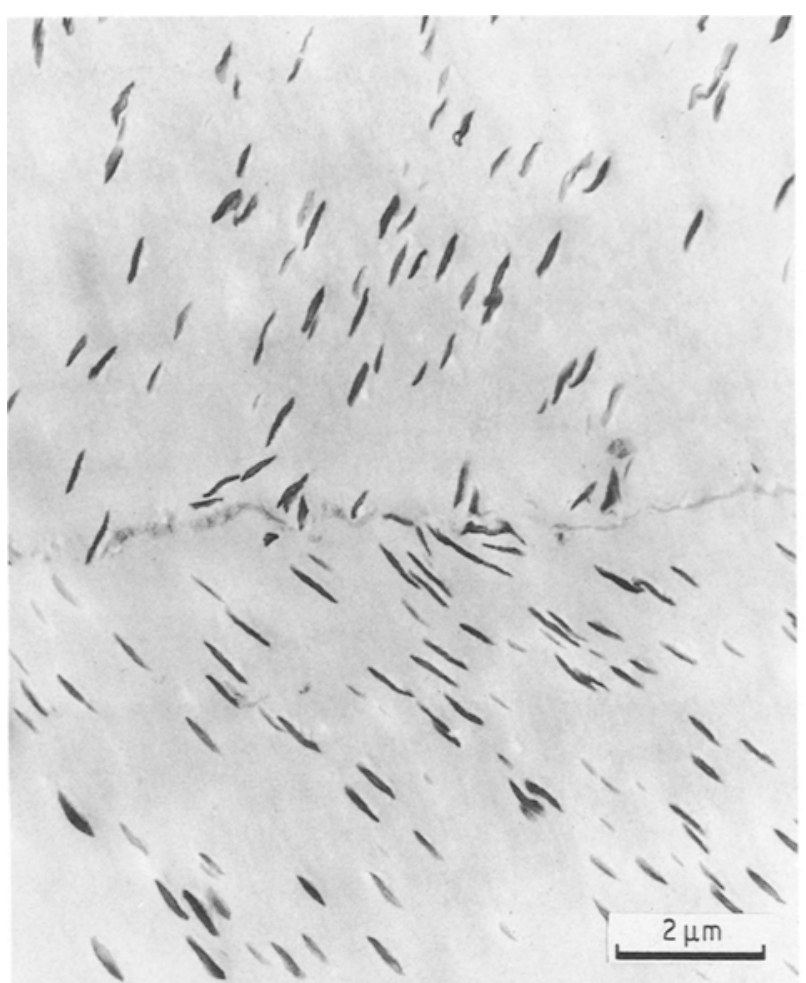

(a)

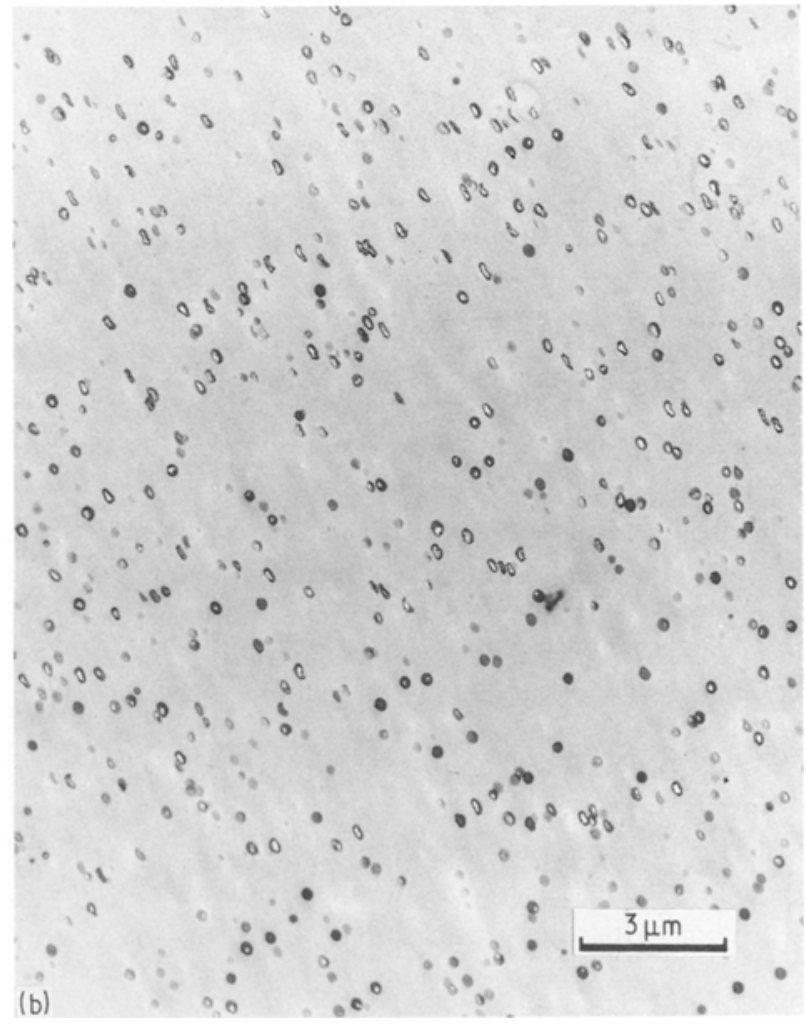

Figure 7 TEM micrographs taken (a) in the DN-4PB crack wake of the PC-KM 653 system and (b) beneath the crack wake. Large elongation and cavitation of the rubber particles are observed. The crack propagates from right to left.

must be within about $10 \%$ to ensure subcritical growth of the surviving crack $[22,27]$.

The distance between the two near identical cracks also needs to be large enough to ensure that the two cracks do not interact with each other. The work conducted by Kanazawa et al. [28] and Sue [27] 


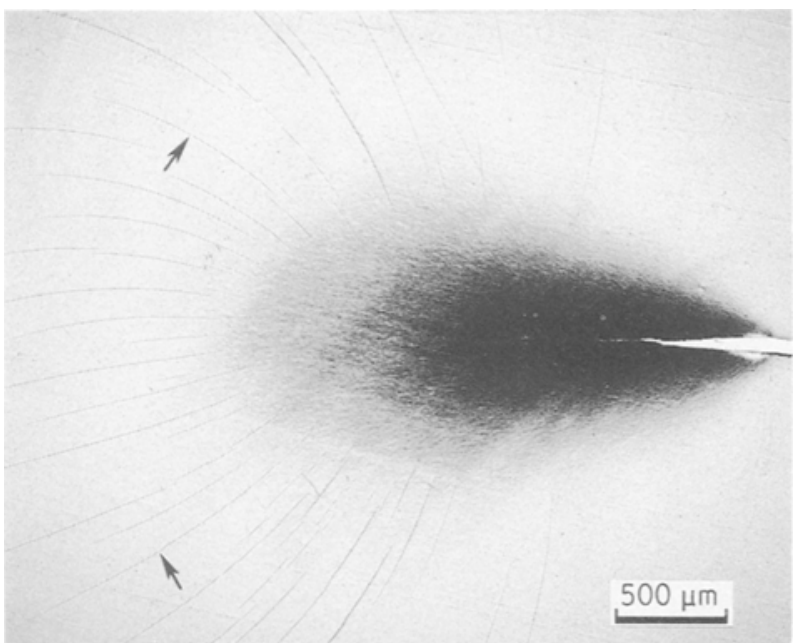

Figure 8 A transmitted-light micrograph of $\mathrm{PC}-\mathrm{KM} 330$ under a dynamic loading condition. Radial cracks (see arrows) can be readily observed. The crack propagates from right to left.

indicates that as long as the ratio between the spacing and length of the two notches is greater than 0.75 for brittle materials, interaction between the two cracks is minimal. In the case of ductile polymers, in order to be certain that the two notches do not interact with each other, it is essential that one investigates the size and shape of the process zones. If the process zones are symmetrical with respect to each crack plane, and the process zones generated by the two cracks do not overlap, then the formation of the SFSZ is likely to be independent of the neighbouring fractured-through crack. One could then be certain that the process zone formed is the natural result of subcritical crack growth. The failure mechanisms obtained from the SFSZ will then be a true representation of crack evolution.

Finally, as pointed out earlier, owing to the nature of the DN-4PB set-up, a subcritically propagated crack is guaranteed to form so long as the two nearidentical cracks are prepared properly. Furthermore, it has been shown that if the toughened plastics are relatively brittle $\left(K_{\mathrm{Ic}} \leqslant 1.2 \mathrm{MPa} \mathrm{m}^{0.5}\right)$, the DN-4PB test can also provide reliable fracture toughness measurements of the toughened system. As a result, the $\mathrm{DN}-4 \mathrm{~PB}$ technique is extremely valuable for those situations where the quantity of the toughened system is limited. This usually happens in the research and development arena.

\section{Conclusion}

The DN-4PB method is shown to be a powerful technique which can reveal the path of evolution of the process (damage) zones. This new insight into the formation of the process zones has revealed complex sequences of toughening mechanisms in toughened polymers. Clearly, this technique complements conventional approaches which often focus on postmortem analyses of fracture surfaces.

Even though the DN-4PB method has been applied to the study of toughened plastics, it should be obvious from the foregoing that the technique is equally applicable to the study of filled polymers such as short-fibre-reinforced plastics.

\section{Acknowledgements}

The authors would like to thank R. A. Pearson and J. Huang for their discussion and E. I. Garcia-Meitin of the Dow Chemical Co. (Freeport, Texas) for the TEM work. This work was supported by a grant from NSF (Material Research Group DMR-8708405).

\section{References}

1. A. F. YEE and R. A. PEARSON, J. Mater. Sci. 21 (1986) 2462.

2. R. A. PEARSON and A. F. YEE, ibid. 21 (1986) 2475.

3. Idem, ibid. 24 (1989) 2571.

4. H.-J. SUE, R. A. PEARSON and A, F. YEE, in "Fracture and Toughening Mechanisms in Polymer Alloys", Proceedings of AICHE 2nd Topical Conference, San Francisco, November 1989.

5. D. S. PARKER, H.-J. SUE, J. HUANG and A. F. YEE, Polymer 31 (1990) 2267.

6. C. B. BUCKNALL, "Toughened Plastics" (Applied Science, London, 1977) p. 195.

7. W. BASCOM, R. TING, R. J. MOULTON, C. K. RIEW and A. R. SIEBERT, J. Mater. Sci. 16 (1981) 2657.

8. J. SULTAN and F. McGARRY, Polym. Eng. Sci. 13 (1973) 19.

9. S. KUNZ-DOUGLASS, P. W..R. BEAUMONT and 'M. F. ASHBY, J. Mater. Sci. 15 (1980) 1109.

10. S. WU, Polymer 26 (1985) 1855.

11. A. G. EVANS, Z. B. AHMAD, D. G. GILBERT and P. W. R. BEAUMONT, Acta Metall. 34 (1986) 79.

12. A. J. KINLOCH, S. J. SHAW, D. A. TOD and D. L. HUNSTON, Polymer 24 (1983) 1341.

13. R. J. M. BORGGREVE, R. J. GAYMANS, J. SCHUIJER and J. F. INGEN HOUSZ, ibid. 28 (1987) 1489.

14. H. BREUER, F. HAAF and J. STABENOW, J. Macromol. Sci.-Phys. B14 (1977) 387.

15. F. J. GUILD and R. J. YOUNG, J. Mater. Sci. 24 (1989) 2454.

16. S. Y. HOBBS and M. E. J. DEKKERS, ibid. 24 (1989) 1316.

17. Z. B. AHMAD, D. G. GILBERT and P.W. R. BEAUMONT, Acta Metall. 34 (1986) 79.

18. H. - J. SUE and A. F. YEE, J. Mater. Sci. 24 (1989) 1447.

19. Idem., ibid. 26 (1991) 3449.

20. H.-J. SUE, PhD thesis, University of Michigan (1988)

21. Idem., Polym. Eng. Sci. 31 (1991) 275

22. Idem., ibid. 31 (1991) 270

23. A. F. YEE, Polymer Materials Sci. \& Eng., American Chemical Soc., 63 (1990) 286.

24. P. C. YANG, E. P. WOO, H.-J. SUE, M. T. BISHOP and D. M. PICKELMAN, ibid. 63 (1990) 315.

25. "O. L. TOWERS, "Stress Intensity Factor, Compliance, and Elastic $\eta$ Factors for Six Geometries" The Welding Institute, Cambridge, England, 1981.

26. J. Lilley and D. G. HOlloway, Philos. Mag. 28 (1973) 215.

27. H.-J. SUE, unpublished work (1990).

28. T. KANAZAWA, S. MACHIDA, S. MOMOTA and Y HAGIWARA, in "Fracture", Proceedings of 2nd International Conference, Brighton, England, Vol. 1 (1969).

Received 27 March

and accepted 17 November 1992 